\title{
Autonomic Nervous System under Ketamine/ xylazine and Pentobarbital Anaesthesia in a Wistar Rat Model: A Chronobiological View
}

\author{
Švorc P. Jr. ${ }^{1}$, Bačová $I^{2}{ }^{2}$, Švorc P. ${ }^{2}$, Bužga M. ${ }^{1}$ \\ ${ }^{1}$ Department of Physiology, Faculty of Medicine, University of Ostrava, Ostrava, \\ Czech Republic; \\ 2Department of Physiology, Faculty of Medicine, Pavol Jozef Šafárik University, \\ Košice, Slovak Republic
}

Received October 31, 2012; Accepted April 8, 2013.

Key words: Anaesthesia - Interval - Ketamine/xylazine - Pentobarbital - Rat Heart rate variability

Abstract: The aim of the present study was to determine the effect of ketamine/ xylazine and pentobarbital anaesthesia on heart rate variability as a marker of autonomic nervous system activity. The experiments were performed in ketamine/ xylazine $(10 \mathrm{mg} / \mathrm{kg} / 15 \mathrm{mg} / \mathrm{kg}$ ) and pentobarbital $(40 \mathrm{mg} / \mathrm{kg}$, i.p.) anaesthetized female Wistar rats, after adaptation to a light-dark cycle of 12 hours light: 12 hours dark. Heart rate variability parameters (RR interval, power VLF (very low frequency), power LF (low frequency), power HF (high frequency) and relative powers) were evaluated during spontaneous breathing as a function of the light-dark cycle (LD cycle). Significant LD differences were found in the duration of RR intervals in ketamine/xylazine compared with pentobarbital-anaesthetized rats. Correlation analysis revealed moderate dependency between the RR interval duration and HF and LF power parameters in ketamine/xylazine anaesthesia in both light and dark parts of the cycle. In pentobarbital-anaesthetized rats, correlation analysis demonstrated a moderate dependence between RR interval duration and HF and VLF power parameters, but only in the dark part of the LD cycle. Ketamine/xylazine anaesthesia increased parasympathetic activity, and suppressed sympathetic and baroreceptor activity independently of the light-dark cycle. LD differences in RR interval duration were not eliminated. Pentobarbital anaesthesia increased parasympathetic activity, decreased sympathetic and baroreceptor activity, and eliminated LD differences in RR interval duration.

This study was supported by VEGA grant $1 / 0423 / 11$ and by CEEPM 26220120067.

Mailing Address: Assoc. Prof. RNDr. Pavol Švorc Jr., PhD., Department of Physiology, Faculty of Medicine, University of Ostrava, Syllabova 19, 70030 Ostrava, Czech Republic; Mobile Phone: +420 725836 819; e-mail: pavolml.svorc@osu.cz 


\section{Introduction}

Besides humans, almost all animals are exposed to periodic repetitions of light and dark cycles during a 24-hour (circadian) period to which virtually all physiological functions are synchronized. Disturbance of the internal synchronization of rhythms with the periodicity of the external environment may manifest by increased susceptibility to disease. Therefore, design of experimental, in vivo, chronobiological animal models may help reveal some of the relationships between circadian time and biological function, which is sometimes very difficult to study in humans. However, in vivo experiments require the use of appropriate anaesthesia, which should be selected according to their effect on the organism.

Any research investigating aspects of the autonomic nervous system and its extensive influence on internal organs should take into account the effects of the anaesthetic agent used. Intravenous anaesthetic agents can have distinct qualitative and quantitative effects on the peripheral autonomic nervous system. Furthermore, anaesthesia may alter the activity of the sympathetic or parasympathetic divisions of the autonomic nervous system, and its compensatory mechanisms.

Administration of anaesthesia during the daytime is also important and may play a crucial role in in vivo animal experiments. For example, in rats, the duration of sleep that is caused by anaesthetic agents such as pentobarbital, hexobarbital and others, is dependent on the state of the circadian system. Potentiated hypnotic effects are likely the result of changes in the daytime sensitivity of the central nervous system to ketamine in the active phase of the circadian cycle (Sato et al., 2004). In mice that were active between 19:00 $\mathrm{h}$ and 01:00 h, and less active between 07:00 $\mathrm{h}$ and 19:00 h, it was found that after the administration of ketamine and pentobarbital at 22:00 h (active phase), significantly more episodes of the loss of righting reflex occurred compared with the administration at 10:00 $\mathrm{h}$ (inactive phase) (Sato et al., 2005).

This particular chronobiological phenomenon can be a problem (Sato et al., 2005) because most reports to date have not described the clock time at which the experiments were performed or the synchronization of the animals to a lightdark (LD) regimen. Although many studies have investigated the factors responsible for changes in specific parameters focusing only on short-term mechanical or metabolic changes, they often do so irrespective of the functional state of the circadian system within a 24 -hour period. This may be a problem because the LD cycle is the strongest synchronizer of endogenous rhythms in experimental animals.

The aim of the present study was chronobiological view on the current state of autonomic nervous system activity in ketamine/xylazine and pentobarbital anaesthesia in spontaneously breathing rats. 


\section{Material and Methods}

Ethics approval

The present study conformed to the Guide for the Care and Use of Laboratory Animals published by the US National Institutes of Health (NIH publication number $85-23$, revised 1996). The study protocol was also approved by the Ethics Committee of the Faculty of Medicine of Pavol Jozef Šafárik University (Košice, Slovak Republic) (permission number 2/05).

\section{Anaesthesia and adaptation of animals}

The experiments were performed in ketamine/xylazine- and pentobarbitalanaesthetized female Wistar rats (ketamine [Narkamon] $100 \mathrm{mg} / \mathrm{kg}$ [SPOFA Prague] + xylazine [Rometar] $15 \mathrm{mg} / \mathrm{kg}$, i.m., pentobarbital [SPOFA Prague] $40 \mathrm{mg} / \mathrm{kg}$, i.p.). Anaesthesia was maintained at a level such that painful stimuli and surgery did not evoke noticeable motor or cardiovascular responses. Anaesthetic condition remained unchanged throughout the study period. On completion of the experiments, rats were sacrificed by cardiac administration of an overdose of ketamine. The effect of the light period on heart rate variability (HRV) was followed after adaptation to an LD cycle ( $12 \mathrm{~h}$ light, $12 \mathrm{~h}$ dark, 40-60\% humidity, room temperature $24^{\circ} \mathrm{C}$, two animals/cage with access to food and water ad libitum) for four weeks, with the dark part of the cycle from 18:00 $\mathrm{h}$ to 06:00 $\mathrm{h}$. The effect of the dark period was followed after adaptation to the inverse setting of the LD cycle (12 $\mathrm{h}$ dark, $12 \mathrm{~h}$ light), with the dark period from 06:00 $\mathrm{h}$ to 18:00 $\mathrm{h}$. The experiments were performed once in each animal in the course of a single LD period (the first animal between 09:00 and 10:00 $\mathrm{h}$ and the second animal between 12:00 and 13:00 h).

\section{Experimental protocol}

Animals were randomly divided into four groups. Two groups were under ketamine/xylazine anaesthesia (light $n=52$ animals, dark $n=27$ animals), and two groups were under pentobarbital anaesthesia (light $n=15$ animals, dark $n=21$ animals) for light and dark periods to evaluate heart rate variability (HRV). HRV measurements were performed during spontaneous breathing in the supine position. The HRV measurements were performed at rectal temperature, which it was maintained by an infrared lamp at a level equivalent to the rectal temperature measured before anaesthetic agent administration.

\section{Measurement of heart rate variability}

Bipolar electrodes were attached to the upper and lower limbs to record an ECG, which was required for analysis of HRV using computer software (Varia Pulse TF4, Sima, Olomouc, Czech Republic). Analysis of HRV was performed by scoring $600 \mathrm{RR}$ intervals needed for calculation of HRV parameters. The following HRV parameters were measured: RR interval duration (ms), very low frequency (VLF) power (corresponding to sympathetic activity), low frequency (LF) power 
(baroreceptor activity, or sympathetic and parasympathetic activity together), high-frequency (HF) power (corresponding to parasympathetic activity) $\left(\mathrm{ms}^{2}\right)$ and relative VLF power, relative LF power and relative HF power (\%).

\section{Statistical analyses}

Data are presented as mean $\pm S D$, and $p<0.05$ was considered to be statistically significant by the parametric $t$-test. The relationship between the evaluated parameters was determined by calculating correlation coefficients. The interval $-0.4>r>+0.4$ was considered to be significant. The data were processed from trials that were conducted independent of the season because circannual variation can also occur in the parameters that were examined.

\section{Limitations of study}

Absence of the HRV parameters (also literary current data) from animals without anaesthesia and in the LD dependence can be a limitation in study. The next limitation can be that HRV was not corrected to the rhythm of spontaneous breathing and a relatively large dispersion of the measured values. The HRV values showed intra- and interindividual variability, which is a problem, mainly concerning in vivo studies. The discrepancy can be explained by the production of spontaneous, unpredictable alterations in the electrophysiological properties of the heart induced by anaesthesia, or hormonal and homeostatic reflexes in the animals.

\section{Results}

Rectal temperature, measured before application of both anaesthetic agents, was $36.3 \pm 0.52{ }^{\circ} \mathrm{C}$ in the light and $37.0 \pm 0.43^{\circ} \mathrm{C}$ in the dark part of the rat regime day. The both anaesthetic agents identically decreased the rectal temperature approximately about $1.5-2.0^{\circ} \mathrm{C}$ (light $34.6 \pm 1.03$; dark $35.6 \pm 0.74$ ). The significant $(p<0.001)$ LD differences were preserved not only before but also after both anaesthetic agent application.

In the ketamine/xylazine-anaesthetized rats, RR interval duration was significantly longer in the light part of the day compared with the dark part (light $0.262 \pm 0.03 \mathrm{~ms}$ vs. dark $0.215 \pm 0.02 \mathrm{~ms}, \mathrm{p}<0.001)$. LD differences were not eliminated. Although LD differences were eliminated in pentobarbital-anaesthetized rats, insignificantly higher values were observed in the light part of the day (light $0.175 \pm 0.01 \mathrm{~ms}$ vs. dark $0.164 \pm 0.02 \mathrm{~ms})$. Significant differences $(p<0.001)$ in RR interval duration between ketamine/xylazine and pentobarbital anaesthesia were found in both lighted parts of the day.

HF power and VLF power showed LD differences in both ketamine/xylazine- and pentobarbital-anaesthetized rats. LD difference was eliminated only in LF power in pentobarbital-anaesthetized rats (Table 1).

The relative powers of individual HRV parameters (Table 2) correspond to the dominant influence of the parasympathetic division of the autonomic nervous 
system, with subtle influences from baroreceptor and sympathetic activities under both ketamine/xylazine and pentobarbital anaesthesia independently of the LD cycle. LD dependence was observed only for r.p. VLF (relative power VLF) with higher sympathetic activity in pentobarbital-anaesthetized rats in the light part of the day. Baroreceptor activity was the same in both the light and the dark parts of the regimen.

Table 1 - Mean values \pm SD of HF, LF and VLF powers in single lighted parts of the day in ketamine/xylazine- and pentobarbital-anaesthetized rats

\begin{tabular}{lccc}
\hline & $\begin{array}{c}\text { HF power } \\
\left(\mathrm{ms}^{2}\right)\end{array}$ & $\begin{array}{c}\text { LF power } \\
\left(\mathrm{ms}^{2}\right)\end{array}$ & $\begin{array}{c}\text { VLF power } \\
\left(\mathrm{ms}^{2}\right)\end{array}$ \\
\hline Ketamine/xylazine & & & \\
\hline light & $5.537 \pm 2.34$ & $0.182 \pm 0.01$ & $0.091 \pm 0.04$ \\
dark & $1.324 \pm 0.59$ & $0.107 \pm 0.06$ & $0.045 \pm 0.02$ \\
$\mathrm{p}<$ & $\mathbf{0 . 0 0 1}$ & $\mathbf{0 . 0 0 1}$ & $\mathbf{0 . 0 0 1}$ \\
\hline Pentobarbital & & & \\
\hline light & $0.529 \pm 0.24$ & $0.192 \pm 0.08$ & $0.273 \pm 0.13$ \\
dark & $1.037 \pm 0.48$ & $0.032 \pm 0.01$ & $0.025 \pm 0.01$ \\
$\mathrm{p}<$ & $\mathbf{0 . 0 5}$ & $\mathbf{0 . 0 0 1}$ & \\
\hline
\end{tabular}

HRV parameters were calculated from 600 RR intervals using computer software Varia Pulse TF4 $\mathrm{p}<$ statistical significant LD differences

Table 2 - Relative powers of HRV in single lighted part of the day

\begin{tabular}{lrrrr}
\hline & \multicolumn{2}{c}{ Ketamine/xylazine } & \multicolumn{2}{c}{ Pentobarbital } \\
& light & dark & light & dark \\
\hline r.p. HF & $86 \%$ & $85 \%$ & $74 \%$ & $84 \%$ \\
r.p. LF & $9 \%$ & $8 \%$ & $12 \%$ & $12 \%$ \\
r.p.VLF & $5 \%$ & $7 \%$ & $14 \%$ & $4 \%$ \\
\hline
\end{tabular}

Parameters were calculated from total power, and expressed as \% under ketamine/xylazine and pentobarbital anaesthesia from the light and dark parts of the light-dark regimen

r.p. HF - relative power HF; r.p. LF - relative power LF; r.p.VLF - relative power VLF

Table 3 - Correlation coefficients between RR interval duration and single parameters of HRV in single lighted part of the day

\begin{tabular}{llccc}
\hline & \multicolumn{2}{c}{ Ketamine/xylazine } & \multicolumn{2}{c}{ Pentobarbital } \\
& light & dark & light & dark \\
\hline RR-HF & $\mathbf{r}=\mathbf{0 . 4 8}$ & $\mathbf{r = 0 . 4 4}$ & $r=0.09$ & $\mathbf{r = 0 . 4 2}$ \\
RR-LF & $\mathbf{r}=\mathbf{0 . 4 6}$ & $\mathbf{r}=\mathbf{0 . 4 5}$ & $r=0.23$ & $r=0.06$ \\
RR-VLF & $r=0.19$ & $r=0.14$ & $r=0.26$ & $\mathbf{r = 0 . 4 7}$ \\
\hline
\end{tabular}

Švorc P. Jr.; Bačová I.; Švorc P.; Bužga M. 
Analysis revealed a moderately positive correlation (light $r=0.48$; dark $r=0.44$ ) between the RR interval duration and HF power in ketamine/xylazineanaesthetized rats in both light and dark parts of the day (Table 3). A similar moderate dependence was observed for LF power (dark $r=0.45$; light $r=0.46$ ). No correlations were found between VLF power and RR interval duration in either the light or dark part of the day. In pentobarbital-anaesthetized rats, moderate correlation dependence was detected for HF, VLF and RR interval duration only in the dark of rat regime day.

\section{Discussion}

In our experimental model, two different results were observed with respect to the preservation of $L D$ differences in RR interval duration during spontaneous breathing with the use of two different anaesthetic agents. In ketamine/xylazineanaesthetized rats, significant LD differences were preserved, in contrast to pentobarbital-anaesthetized rats. Although the disruptive effect of ketamine on circadian rhythms has been described by others (Prudian et al., 1997; Pelissier et al., 1998), this effect was associated with modification of acrophase, amplitude or mesor, but without loss of daily rhythmicity. Similar, but probably more pronounced rhythm alterations occur in rats anaesthetized with pentobarbital. Other explanations for the modulation of daily rhythmicity may be found in the studies by Mortola and Seifert (2000), Bishop et al. (2001), Bosco et al. (2003), Kaplan et al. (2003) and Mortola (2007). The fact that hypoxia modifies the circadian oscillation of important variables, such as body temperature and metabolism, may lead to the expectation that the daily rhythms of many functions are disrupted by hypoxia according to their relationships and connection with the primary variables. This hypoxic state likely contributes a larger parasympathetic influence on the heart (Hayashida et al., 1996).

The parasympathetic influence on the heart was described by other authors. Ketamine/xylazine anaesthesia reduces heart rate in isolated rat heart preparations (Aronson and Hanno, 1978) and also in in vivo conditions (Salerno and van Tienhoven, 1976; Sapru and Krieger, 1979; Brown et al., 1994; Hoque et al., 1996; Maignan et al., 2000). It prolongs RR and QT intervals (Aronson and Hanno, 1978), decreases blood pressure (Sapru and Krieger, 1979), renal sympathetic activity and attenuates baroreflex sensitivity in vivo (Akine et al., 2001). On the other hand, Reid et al. (2003) described a stimulating effect on cardiac function during resuscitation. Unfortunately, in the above-mentioned studies, the clock time of the experiment was not reported, nor was the synchronization of animals to an LD regimen described.

The effect of pentobarbital on the cardiovascular and autonomic nervous system has been studied by others. It is suggested that the parasympathetic nervous system is primarily responsible for bradycardia, which is caused by the baroreceptor reflex in conscious rats. The sympathetic and parasympathetic system 
also contribute to the control of tachycardia induced by baroreceptor reflex, except in extreme cases of acute hypotension, in which tachycardia is preferably evoked by the activation of sympathetic nerves. Pentobarbital has been shown to diminish the tachycardia response, reduce blood pressure and directly affect the sympathetic nervous system in rats (Shimokawa et al., 1998). Mild hypotension and tachycardia in rats were observed with the bolus injection of pentobarbital $(5,10$ and $20 \mathrm{mg} / \mathrm{kg})$ in a dose-dependent manner (Watkins and Maixner, 1991). In a study involving pentobarbital-anaesthetized rats, Hoque et al. (1996) reported a significant decrease in mean blood pressure, but without any tachycardia. Pentobarbital anaesthesia was associated with statistically significant reductions in the release of norepinephrine and the metabolic rate of norepinephrine clearance, whereas plasma levels of norepinephrine were not changed. Pentobarbital induced a decrease in blood pressure with a transient decrease in heart rate, and did not alter renal sympathetic activity, but decreased baroreflex activity (Sato et al., 2005). Pentobarbital has been shown to slightly suppress protein synthesis in the brain, and also suppresses cardiac and respiratory function during resuscitation (Reid et al., 2003).

Watkins and Maixner (1991) studied the impact of pentobarbital anaesthesia on the control of the autonomic nervous system with reflex bradycardia mediated by baroreceptors. In awake and anaesthetized rats, pentobarbital induced hypotensionmediated reflex bradycardia after administration of phenylephrine before and after sympathetic blockade with ethanol or beta-1-receptor antagonists, or parasympathetic blockade with muscarinic receptor antagonist methylatropines. Reflex bradycardia has been shown to be significantly reduced by pentobarbital anaesthesia; cardio-sympathetic blockade caused an equivalent decrease in baroreflex activity. Conversely, blockade of the parasympathetic nervous system with methylatropine caused relatively lower inhibition of baroreflex activity in pentobarbital-anaesthetized rats compared with awake rats. These results suggest that pentobarbital anaesthesia reduces baroreflex activity by inhibiting the reflex bradycardia mediated by vagal activation.

The above results demonstrate that pentobarbital anaesthesia can reduce baroreflex activity or diminish tachycardia which, according to some authors, does not influence heart rate. If the reference range of heart rate in the rat is considered to be between 260 and 450 beats/min (Hess et al., 1984), no heart rate changes were observed in our experimental model. Pentobarbital only eliminated LD differences (light $343 \pm 28$ beats $/ \mathrm{min}$, dark $365 \pm 44$ beats $/ \mathrm{min}$ ). Results showed that pentobarbital anaesthesia acts on the autonomic nervous system in a manner that is dependent on the LD cycle. Although the parasympathetic activity exceeded in the both the light and dark parts, the sympathetic activity was significantly increased in the light compared with the dark. This fact may explain the loss of LD differences in the RR interval duration. It is interesting that neither the sympathetic or parasympathetic, nor baroreceptor activity had any influence on the RR interval duration during the light cycle. In the dark part of the cycle, the control of the RR 
interval duration was mediated by parasympathetic as well as sympathetic drives (without participation of the baroreceptor activity).

\section{Conclusion}

Results demonstrate that ketamine/xylazine anaesthesia preferably increases parasympathetic activity and suppresses sympathetic and baroreceptor activity, resulting in marked bradycardia independently of the LD cycle. Presence of LD differences refers to the fact that this type of anaesthesia may be applicable in chronobiological studies. However, it may be problematic for cardiovascular research owing to serious bradycardia.

Pentobarbital anaesthesia increases parasympathetic activity and decreases sympathetic and baroreceptor activity by a manner dependent on the LD cycle. The loss or reduction of LD differences refers to the fact that this type of anaesthesia may be applicable in cardiovascular experiments, but not in chronobiological studies because it modifies the daily rhythmicity of the autonomic nervous system activity.

\section{References}

Akine, A., Suzuka, H., Hayashida, Y., Kato, Y. (2001) Effects of ketamine and propofol on autonomic cardiovascular function in chronically instrumented rats. Auton. Neurosci. 87(2-3), 201-208.

Aronson, C. E., Hanno, E. R. S. (1978) Effects of ketamine on the isolated perfused rat heart. Gen. Pharmacol. 9(4), 249-255.

Bishop, B., Silva, G., Krasney, J., Nakano, H., Roberts, A., Farkas, G., Rifkin, D., Shucard, D. (2001) Ambient temperature modulates hypoxic-induced changes in rat body temperature and activity differentially. Am.J. Physiol. 280(4), R1190-R1196.

Bosco, G., lonadi, A., Panico, S., Faralli, F., Gagliardi, R., Data, P., Mortola, J. P. (2003) Effects of hypoxia on the circadian patterns in men. High Alt. Med. Biol. 4, 305-318.

Brown, D. R., Brown, L. V., Patwardhan, A., Randall, D. C. (1994) Sympathetic activity and blood-pressure are tightly coupled at $0.4 \mathrm{~Hz}$ in conscious rats. Am. J. Physiol. 267(5), 1378-1384.

Hayashida, Y., Hirakawa, H., Nakamura, T., Maeda, M. (1996) Chemoreceptors in autonomic responses to hypoxia in conscious rats. Adv. Exp. Med. Biol. 410, 439-442.

Hess, L., Dvořáček, I., Svobodník, J. (1984) Anestézie Laboratorních Zviřrat (Anaesthesia of Laboratory Animals). Avicenum, Praha. (in Czech)

Hoque, A. M., Marczin, N., Catravas, J. D., Fuchs, L. C. (1996) Anesthesia with sodium pentobarbital enhances lipopolysaccharide-induced cardiovascular dysfunction in rats. Shock 6(5), 365-370.

Kaplan, J. L., Gao, E., DeGaravilla, L., Victain, M., Minczak, B., Dalsey, W. C. (2003) Adenosine A1 antagonism attenuates atropine-resistant hypoxic bradycardia in rats. Acad. Emerg. Med. 10, 923-930.

Maignan, E., Dong, W. X., Legrand, M., Safar, M., Cuche, J. L. (2000) Sympathetic activity in the rat: Effects of anaesthesia on noradrenaline kinetics. J. Auton. Nerv. Syst. 80(1-2), 46-51.

Mortola, J. P. (2007) Hypoxia and circadian patterns. Respir. Physiol. Neurobiol. 158, 274-279.

Mortola, J. P., Seifert, E. L. (2000) Hypoxic depression of circadian rhythms in adult rats. J. Appl. Physiol. 88, 365-368.

Pelissier, A. L., Gantenbein, M., Prudian, F., Bruguerolle, B. (1998) Influence of general anaesthetics on circadian rhythms of heart rate, body temperature and locomotor activity in rats. Stal 23(2), 91-98. (in French) 
80) Prague Medical Report / Vol. 114 (2013) No. 2, p. 72-80

Prudian, F., Gantenbein, M., Pelissier, A. L., Attolini, L., Bruguerolle, B. (1997) Daily rhythms of heart rate temperature and locomor activity are modified by anaesthetics in rats: a telemetric study. Naunyn Schmiedebergs Arch. Pharmacol. 355(6), 774-778.

Reid, K. H., Paskitti, M., Guo, S. Z., Schmeizer, T., lyer, V. (2003) Experience with ketamine and sodium pentobarbital as anesthetics in a rat model of cardiac arrest and resuscitation. Resuscitation 57(2), 201-210.

Salerno, A., van Tienhoven, A. (1976) The effect of ketamine on heart rate, respiration rate and EEG of white leghorn hens. Comp. Biochem. Physiol. C 55(1), 69-75.

Sapru, H. N., Krieger, A. J. (1979) Cardiovascular and respiratory effects of some anesthetics in the decerebrate rat. Eur.J. Pharmacol. 53(2), 151-158.

Sato, Y., Kobayashi, E., Hakamata, Y., Kobahashi, M., Wainai, T., Murayama, T., Mishina, M., Seo, N. (2004) Chronopharmacological studies of ketamine in normal and NMDA $\varepsilon 1$ receptor knockout mice. Br.J. Anaesth. 92(6), 859-864.

Sato, Y., Seo, N., Kobahashi, E. (2005) The dosing-time dependent effects of intravenous hypnotics in mice. Anesth. Analg. 101, 1706-1708.

Shimokawa, A., Kunitake, T., Takasaki, M., Kannan, H. (1998) Differential effects of anesthetic on sympathetic nerve activity and arterial baroreceptor reflex in chronically instrumented rats.J. Auton. Nerv. Syst. 72(1), 46-54.

Watkins, L., Maixner, W. (1991) The effect of pentobarbital anesthesia on the autonomic nervous system control of heart rate during baroreceptor activation. J. Auton. Nerv. Syst. 36(2), 107-114.

Švorc P. Jr.; Bačová I.; Švorc P.; Bužga M. 